\title{
Physical activity for children undergoing acute cancer treatment: a qualitative study of parental perspectives
}

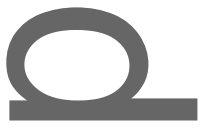

Authors

Sarah L Grimshaw, Bachelor of Physiotherapy

La Trobe University, School of Allied Health, Human Services and Sport, Bundoora, Melbourne, VIC, 3086. Australia.

Murdoch Children's Research Institute, Parkville, Melbourne, VIC, 3052. Australia

Professor Nicholas F Taylor, PhD

La Trobe University, School of Allied Health, Human Services and Sport, Bundoora, Melbourne, VIC, 3086. Australia

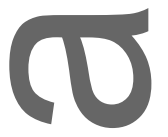

Francoise Mechinatud, M.D.

Unité Hématologie-Immunologie, Hôpital Robert Debré, Paris. France

APHP Nord-Université de Paris, Paris. France

Associate Professor Rachel Conyers, MD, PhD

The Royal Children's Hospital, Children's Cancer Centre, Parkville, Melbourne, VIC, 3052. Australia

This is the "thor manuscript accepted for publication and has undergone full peer review but has not been througn tne copyediting, typesetting, pagination and proofreading process, which may lead to differenc this version and the Version of Record. Please cite this article as doi: $10.1002 / \mathrm{pbc} .28264$.

This article is prot ted by copyright. All rights reserved.

Meeting publio $\mathbf{n}: 21^{\text {st }}$ World Congress of Psycho-oncology

Date: 25 Se tember 2019

Title: Physical activity for children with cancer: the parental perspective

Journal of Psychosocial Oncology Research and Practice: September 2019 - Volume 1 - Issue 1S - p $\underline{\mathrm{e} 10}$

doi: 10.1097/OR9.0000000000000010 
Murdoch Children's Research Institute, Parkville, Melbourne, VIC, 3052. Australia

\section{Professor Nora Shields, PhD}

La Trobe University, School of Allied Health, Human Services and Sport, Bundoora, Melbourne, VIC, 3086. Australia

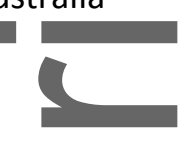

\section{Correspondence}

Sarah L Grimshaw. School of Allied Health Human Services and Sport, La Trobe University, Bundoora VIC 3086. Telephone: +61 39479 5852. Fax: +61 39479 5768. Email: sarah.grimshaw@mcri.edu.au

\section{Word Count}

Abstract: 250

Main Text: 3623 (excluding data in text boxes)

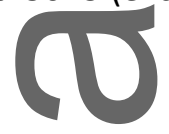

Short running title: Physical activity for children with cancer

Tables: 2

Figures: 1

\section{Supplementary files: 1}

Keywords: Pediatric, child, cancer, exercise, physical activity, qualitative, parents

\begin{tabular}{l} 
Abbreviations: \\
\begin{tabular}{|l|l|}
\hline ANUM & Associate nurse unit manager \\
\hline NS & Nora Shields \\
\hline SG & Sarah Grimshaw \\
\hline y/o & Year old \\
\hline
\end{tabular} \\
\hline
\end{tabular}




\section{ABSTRACT}

Background. Little is known about how to facilitate participation in physical activity among children receiving acute cancer treatment.

Objective. To understand the parental perspectives on physical activity for children during acute cancer treatment and explore strategies to overcome physical inactivity.

Methods. Aqualitative study was completed. Data were collected via semi-structured interviews with parents of children (aged 4-18 years) who were in their first nine months of cancer treatment. Data were analysed thematically.

Results. Twenty parents were interviewed. A childhood cancer diagnosis and subsequent treatment was described as setting in motion a spiral of physical inactivity. Parents identified movement restrictions as a result of commencing treatment and the hospital environment as factors initiating this decline. Parents described the subsequent impact of movement restrictions on their child over time including: loss of independence, isolation and low motivation. These three consequences further contributed to an inability and unwillingness to be physically active. Parents responded in a variety of ways to their child's inactivity, many were motivated to overcome the barriers to physical activity yetexhibited a reduced capacity to do so. Suggested intervention strategies highlighted the need for comprehensive support from the organisation providing treatment.

Conclusions. Reasons for reduced physical activity in children receiving acute treatment for cancer are complex and multifactorial. Inactivity cannot be addressed by children and parents alone but requires support from the oncology team through changes to the environment, services and policies to promote physical activity. These findings may be used to inform targeted, effective and feasible physical activity interventions.

\section{INTRODUCTION}

Children with cancer have reduced physical activity levels during treatment compared to their peers. ${ }^{1-3}$ Children can also experience a range of adverse treatment effects that compromise their physical function. ${ }^{2,-7}$ These adverse effects, in addition to reduced levels of physical activity, have implications for their development and their health and well-being. If not addressed, functional deficits can worsen over time. Increasing levels of physical activity during acute cancer treatment may help to minimise or prevent adverse effects for children in the short and long-term. ${ }^{8}$

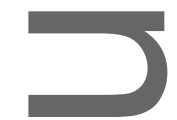

There is growing evidence to support the feasibility and effectiveness of physical activity interventions in the acute treatment setting. ${ }^{9-11}$ Despite this, high drop-out rates and poor compliance are commonly reported as limiting factors. ${ }^{10,12-15}$ To implement effective physical activity interventions we need a deeper understanding of how to engage children in physical activity in the acute cancer treatment setting. Previous studies have investigated barriers and motivators to physical activity from the child's perspective. ${ }^{16,17}$ In these studies, children discussed the influence 
parents have on their physical activity participation, highlighting the importance of understanding parents' perspectives. ${ }^{18}$

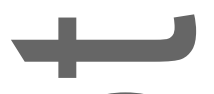

Parents could be key players in promoting increased activity levels in children with cancer. Qualitative studies in childhood cardiac and obesity populations have found parental values and attitudes to physical activity predict a child's physical activity behaviour. ${ }^{19,20}$ In the cancer setting, mothers of children with cancer have a strong protective role and are integral in ensuring adherence to medical treatment to maximise their child's well-being. ${ }^{21}$ It is unclear what beliefs, values and knowledge parents of children with cancer have of physical activity and how this may dictate their child's behaviourduring and beyond treatment. There is also a lack of understanding about how parents respond to their child's inactivity, and the barriers and motivators towards physical activity from their perspective. Qualitative methods preface the description and understanding of people's experiences, emotions and behaviours and is an effective means of addressing this current gap. ${ }^{22}$ Understanding the parental experience, and the roles they play, could help to identify educational needs and new motivators toward physical activity for children with cancer. Consistent with implementation research methods, this qualitative inquiry is an important step toward designing feasible physical activity interventions in the cancer treatment setting ${ }^{23}$.

Our primary aim was to understand parental perspectives on physical activity for children during acute cancer treatment. The secondary aim was to explore parental perceptions of what strategies may be helpfut in encouraging children to be more physically active.

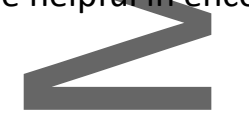

\section{METHODS}

\section{Design}

This qualitative study was designed from a constructivist perspective, which aims to build new understanding of the constructions participants (and researchers) have in regard to the research questions. This framework acknowledges participant opinions and experiences are varied and complex, and that multiple "knowledges" exist. Data were analysed inductively, with meaning generated from data collected. ${ }^{24}$

\section{Population}

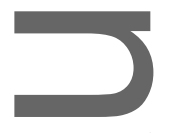

Ethics approval was obtained prior to study commencement. Participants were recruited from a large tertiary paediatric hospital in Melbourne, Australia between 1 August 2017 and 31 January 2018. Written informed consent was obtained from all participants. A purposive sampling strategy was used. ${ }^{22}$ Eligibility criteria are presented in Table 1 . Sampling aimed to maximise diversity across child characteristics including age, diagnosis, functional ability, time since diagnosis, treatment modality, time spent as an in-patient and place of residence. Parents were not contacted within the 
first month of diagnosis as this is a time of high stress for families. Participation was limited to parents of children diagnosed with cancer within the previous nine months, to capture the experiences of those receiving acute treatment. Hospital staff, independent to the research team and trained regarding the details of the study, helped identify eligible parents and approached potential participants.

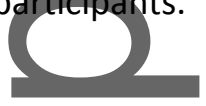

\section{Data collection}

Semi-structured interviews were conducted using an interview guide with open-ended questions and prompts (Supplementary File S1). The lead investigator (SG) completed all interviews. Physical activity was defined as: "any bodily movement produced by skeletal muscles that requires energy expenditure" 25 . Interview prompts were altered during the study in response to emergent themes. Interview timing was sensitive to existing hospital appointments and parents were interviewed faceto-face, by telephone or videoconference. The length of the interview was not time limited to allow for sufficient engagement with participants and thorough discussion of themes. Interviews were recorded with consent and transcribed verbatim.

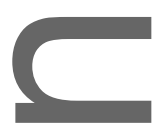

\section{Data analysis}

Thematic analysis was conducted inductively, as described by Braun $^{26}$ and Boyatzis ${ }^{27}$. The first phase of data analysis was familiarisation. Once transcribed, interview transcripts were read through in their entirety to identify emerging patterns. The second phase involved systematic data coding, with codes being data=driven. Computer software (NVivo, Melbourne) was used to assist in data coding. The third phase was thematic development, where coded data were collated into themes. Relationships between themes were considered. The fourth phase involved reviewing, refining and naming themes. Analysis was ongoing throughout the study, recruitment ceased once saturation was achieved.<smiles></smiles>

Data were coded independently by two researchers (SG, NS). Triangulation of data coding between researchers was undertaken to gain deeper insights but also to compare and contrast the subjective views of the researchers. ${ }^{28}$ In line with a constructivist, inductive perspective it was expected that researchers would formulate their own interpretations of the data. ${ }^{29}$ Resultant themes were compared and contrasted and any inconsistencies or deviant cases discussed and reported. Disagreement between the researchers was settled by consensus. ${ }^{30}$ Participants were invited to participate in member checking. Participants were sent a document containing a summary of the resultant major themes and given the opportunity to respond with additional comments if they felt that their views were not adequately reflected. Field notes were kept for reflexive analysis purposes. 


\section{Rigour}

As a physiotherapist working on the cancer ward at a tertiary hospital, the potential for the lead investigator's prior experience and preconceived opinions to influence the results of this study is acknowledged. ${ }^{24,31}$ Effort was made to minimise bias through techniques to ensure rigour. Credibility was strengthened through using purposive sampling, member checking, peer examination, reflexive analysis, data triangulation and transparent presentation of findings. ${ }^{22,32,33}$ Transferability was addressed through clear reporting of the participants, methods and research processes. ${ }^{33}$ To ensure $^{3}$ dependability, transparent descriptions of aims, data gathering, analysis, and interpretation were provided. ${ }^{22}$ An audit trail increased confirmability, assuring readers the resultant findings and interpretations were derived from data and not from opinions and biases of the investigators. ${ }^{22}$

\section{RESULTS}

Parents of 30 children were approached to participate. Five parents declined, 4 were lost to follow up and in one case the child turned 19 years prior to finalising recruitment, leaving 20 participants who participated in the study (see Table 2). Interview length was between 16 and 52 minutes (median 37 minutes). All parents of a child with a diagnosis of a central nervous system tumour approached declined to participate. Recruitment stopped once data were deemed saturated, determined via the use of a coding workbook and data saturation table once no further themes or codes were identified. ${ }^{34,35}$ A further two interviews were carried out beyond this point to confirm saturation. One parent replied as part of the member checking process, the additional information did not provide any further insights.

The parents' experiences described: (1) factors that contribute to a child's physical inactivity during acute cancer treatment, (2) their responses to physical inactivity and (3) their perspectives on overcoming physical inactivity.

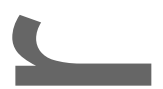

\section{Factors that contribute to physical inactivity}

Based on the emergent themes, a model was developed which describes a 'spiral of physical inactivity' initiated from when a child is first diagnosed with cancer (Figure 1). Factors that contribute to this spiral are represented by the outer and inner circles of the model. The outer circle represents movement restrictions imposed on children as a result of commencing cancer treatment and the hospital environment. The inner circle represents the impact of this restricted movement on a child over time in terms of loss of independence, isolation, and low motivation. An acute medical complication and long-term hospital admission were described as accelerating this spiral.

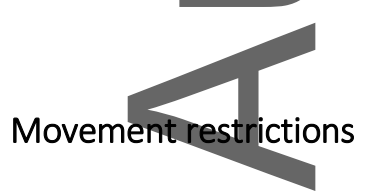

Movement restrictions associated with commencing treatment and the hospital environment were thought to instigate a sudden and rapid decline in a child's ability to be physically active. 


\section{Commencing cancer treatment}

Parents described the impact of commencing treatment as devastating for their child. Treatmentrelated adverse effects such as muscle wasting, weight loss, fatigue, nausea and pain were seen to impede their child's ability to move and participate. Parents described externally imposed restrictions to their child's movement, such as the presence of intravenous lines. Commencing treatment was described as a major disruption to family life, often necessitating relocation from home, a high number of medical appointments, frequent and prolonged hospital admissions. Parents reported these precluded children from participating in physical activities normally part of their daily routine.

"...he's lost muscle mass and lost his fitness, lost the weight, everything...he gets really tired, his fitness, his energy level, his food intake, it's all changed." (Participant 14, father of 9 y/o male with leukemia)

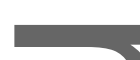

"...we get nervous about the line... it's got the be controllable, you can't just go and play like a child." (Participant 26, mother of 5 (y/o) male with leukemia)

The hospital environment

According to parents, receiving treatment in a hospital environment (both the in-patient and outpatient setting) further reduced their child's ability to move. Parents considered the hospital inadequately resourced to promote physical activity, in terms of: access to activities of daily living, equipment; enticing, age-appropriate facilities, open spaces; and availability of specialised staff. Residing in small spaces and restricted access to outdoor fresh air were of particular concern, especially while admitted to the ward.

"When she's on the ward, no, zero activity, what do you do apart from... walk your drip to the hub. They do nothing, they can't, and there's nothing really they can do," (Participant 12, father of $10 \mathrm{y} / \mathrm{o}$ female with leukemia)

"Something to work towards... some of the them lose the will to live, in terms of not having anything to work towards in here. "(Participant 26, mother of $5 \mathrm{y} / \mathrm{o}$ male with leukemia)

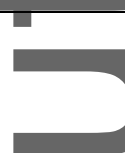

Parents' perceived the priority of the oncology team was medical management, rather than a holistic approach encompassing their child's physical condition. Parents expressed disappointment that physical activity was not a core part of their child's treatment. They described receiving 'generic' and 'inconsistent' advice and that specialised services (such as physiotherapy) was reactive, inadequate or unsuccessful in encouraging physical activity. 


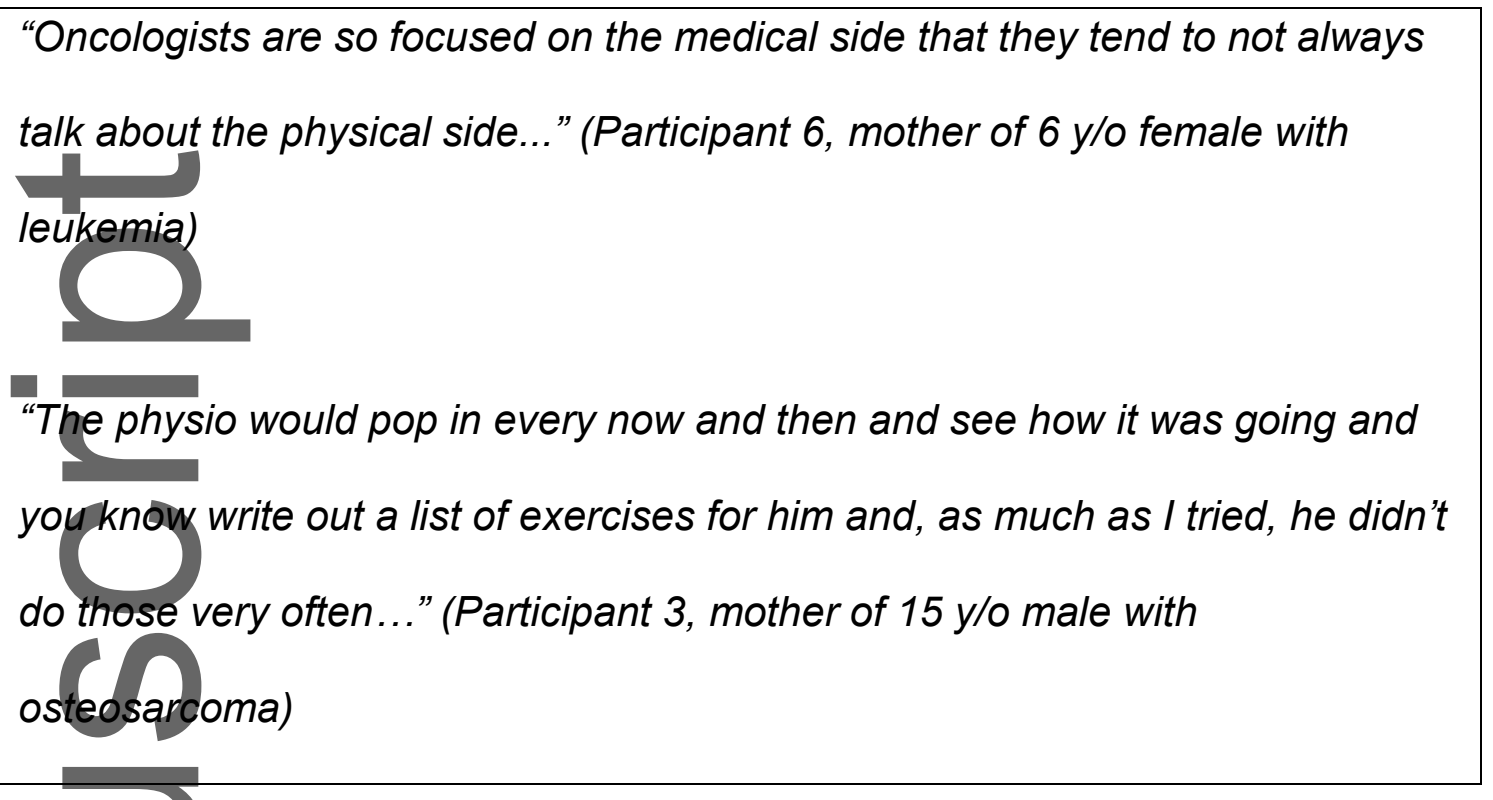

Rules and policies enforced over the course of treatment by the oncology team to promote safety were also perceived as restricting movement. Parents said they understood the reasons for these rules but were frustrated by the inflexible, inconsistent way they were applied by staff. Examples included having to avoid crowded places and reside close to the hospital and prolonged inpatient stays. Restrictive rules specific to the ward environment included: having to remain on the ward while connected to an intravenous pole, isolation due to infection risk and not being allowed to disconnect from their line.

\footnotetext{
"Every time she goes outside, the benefit she's gets from going outside far outweighs the fighting for it, but you will have to fight. You will have to go to the doctors, and then you will have to fight with the ANUM (Associate nurse unit manager) who will then go "no, our policy is that you cannot go outside"'" (Participant 1, mother of 8 y/o female with leukemia)

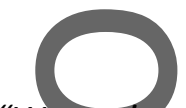

"We can't take her to outside activities, so we are stuck in the city where it is built up...it is also limiting to what you can do and where you can go and what activities she can do." (Participant 12, father of $10 \mathrm{y} / \mathrm{o}$ female with leukemia)"

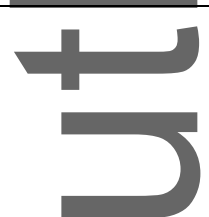

Consequences of restricted movement for children over time The combination of restricted movement, and the protracted nature of cancer treatment, was perceived as contributing to a progressive decline in their child's physical and mental well-being; resulting in lost independence, isolation and low motivation. Parents described these negative consequences as interacting with each other to perpetuate inactivity over time.
} 
Loss of independence

Parents described their children feeling a loss of control over their environment and bodies, including their ability to move, daily routines and the freedom to pursue interests. Parents used language such as 'loss of dignity', 'loss of identity', 'fear' and 'anger' to describe their child's responses to this lack of control and autonomy and talked about how their children grieved these changes. Some children were described as actively refusing physical activity to regain control.

"He really identified himself as a skateboarder and now he feels like he's got
"And mentally he also just kind of refused.... he was just so angry, ... when
he's in hospital, even though the best thing for him is to walk, no, he won't."
(Participant 21, mother of 9 y/o male with lymphoma)

Isolation

Parents described how their child experienced social and physical isolation due to long-term restricted access to open spaces and social environments over the course of acute treatment. In addition, reclusive behaviour was described, especially in adolescents. In the parents' opinion, reclusive behaviour developed due to lost confidence, unstimulating environments and mental health decline. Embarrassment and fear associated with lost ability and changes to physical appearance meant many children and adolescents limited their interactions with the external environment and their peers.

"...he says he hates what he sees in the mirror and he just won't let anyone, apart from family see him." (Participant 30, mother of 17 y/o male with lymphoma)

"There was no way we would, she couldn't get out. And that played a lot of her psyche as well, she felt very, like a prisoner I guess in her own home. And she didn't want to go out either." (Participant 7, mother of $15 \mathrm{y} / \mathrm{o}$ female with leukemia)

Low motivation

Parents talked about the increasing difficulty they experienced in engaging their child in physical activity and their child's growing preference for sedentary activities. Children were perceived to experience a lack of joy with physical activity due to declining ability and reduced access to activities and sporting pursuits that inspired movement, fun and interest. Some children, particularly those 
who identified strongly as being athletic, chose not to take part in physical activity, because they could no longer participate in the sports they love to their previous level of ability.

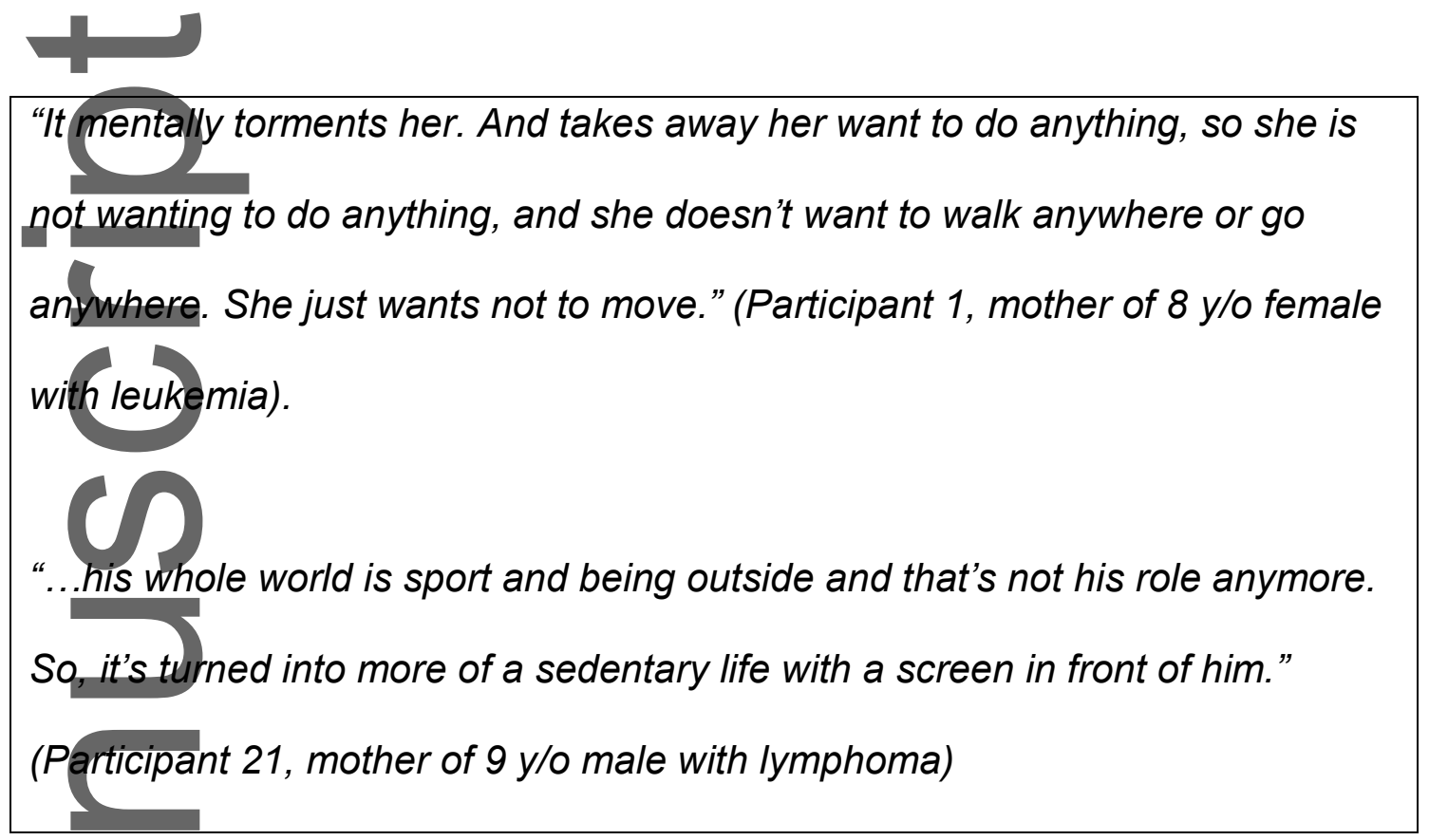

\section{Parental response to physical inactivity}

Parents responded in different ways to their child's decreasing physical activity. Many parents said they tried to take the initiative to promote physical activity and advocated strongly with staff to facilitate it. This was driven by their experience and knowledge about the benefits of physical activity. Despite this, parents felt their ability to effectively promote physical activity was limited. These parents felt activity promotion was solely their responsibility and that they lacked knowledge of how to safely and effectively encourage it in the cancer setting. This was especially true in cases where implementing physical activity was challenging. Parents expressed a sense of hopelessness in their ability to overcome inactivity and how inevitably, they lost motivation over time due to competing demands and responsibilities.

"...we have to try and get him to take meds, we have to get him to bath, we
have to get him to eat, ....and all of those take a lot of energy from us to try
and self-motivate him and enthusiastically get him to participate without him
getting upset." (Participant 26, mother of 5 y/o male with leukemia)
"...so I don't know how we could make it better because the further you go
along and the more treatment you have, the more you can't do." (Participant
29, mother of 12 y/o male with leukemia)


Other parents didn't think to encourage physical activity, despite having positive values towards it. Parents reported feeling so stressed and overwhelmed by the cancer diagnosis that it impeded their ability to attend to anything other than the survival of their child. They also described underestimating the cumulative impact of treatment overtime.

“. you're in so much shock and the child is so young and they're so

distressed and there's just so much mental stuff going on that I don't think we

thought about it unless, you know, the doctors or someone would say

something about physical activity." (Participant 6, mother of 6 y/o female with

leukemia)

)

"I think we all make the mistake of just hanging out waiting for the time that he's better and he can do it all again. I think we're oblivious to the fact of how long it's going to take..." (Participant 21, mother of 9 y/o male with lymphoma)

Some parents believed the benefits of physical activity did not translate to the cancer treatment setting. In these cases, parents didn't encourage activity or actively discouraged through fear of increasing fatigue, infection risk, or weight loss (see Figure 1).

"I didn't want him to move from the bed at the beginning...I didn't want him down because I'd seen him kind of dying and I was like just keep him there until he gets better." (Participant 8 , mother of 4 / / male with leukemia)

\section{Parental perspectives on overcoming physical inactivity}

Parental perspectives were that many of the barriers to physical activity were out of their control and that they, and their child, needed additional support. Suggested interventions targeted their child's unique experience and focused on maximising independence, overcoming isolation and inspiring motivation towards physical activity. There were parents who called for changes to policy and culture within the treatment environment, changes that considered physical activity a vital part of their child's health. More responsive, proactive and consistent therapy services and symptom management was proposed as part of this recommendation.

"Just keep asking to see the physio because I think it's really important that kids actually get that, get that expert help and then that will help them to feel more in 
control, instead of having all that control taken off them." (Participant 4, mother of 16 y/o female with osteosarcoma)

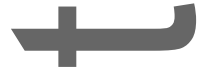

Other parents focused on improvements to the ward environment that included: freely accessible toys, activities, technology and equipment that promoted fun and active play; better access to large and socially interactive spaces; a greater focus on participation in incidental activities as part of daily living and routine; and policy changes that allowed their child to routinely get off the ward and outside forfresh air. Facilitated line-free time was a seen as key to achieving this. Hospital policies and programs that supported children to spend more time at home, away from the hospital environment, was another recommendation.

"If he could've gone downstairs and been allowed to go to the Parkville café
and have his breakfast he would've been down there like a shot." (Participant
10, mother of 17 y/o male with osteosarcoma)
"Whereas if you had a game console that you move around, and you do like
them. Can you do this many steps, or collect, we've been doing it as collect
this amount of ponies for the day." (Participant 1, mother of 8 y/o female with
leukemia)

Parents saw themselves as an underutilised resource. They sought more detailed and repeated education about the importance of physical activity and how to promote it. They also recommended the provision of regular supervised sessions that promoted physical activity with their child and better access to peer support.

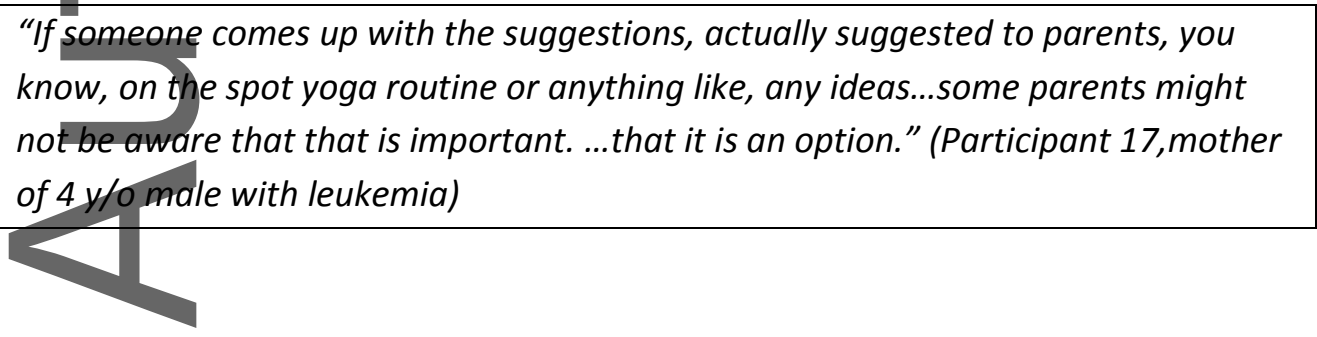




\section{DISCUSSION}

This study adds novel insight into how to design more effective physical activity interventions for children undergoing acute cancer treatment. Parents described a spiral of physical inactivity that follows a diagnosis of childhood cancer, highlighting the interplay between a child's ability to keep physically active, adverse treatment effects, hospital environments and compromised physical and mental health. Parents suggested strategies to increase participation in physical activity; emphasising a need for additional services, support and engagement at an organisational level. Based on these findings, physical activity interventions need to target multiple levels of change across the physical environment, the family and the child; thereby indicating the need for theoryinformed complex intervention design. ${ }^{36}$ The social-ecological model assumes the necessity of multiple levels of influence in order to bring about behaviour change; including intrapersonal, interpersonal, and organisational factors and can be used a framework to apply these results. ${ }^{37}$

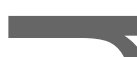

Interventions at an intrapersonal level address characteristics of the individual; including their knowledge, attitudes, skills and intentions in the context of behaviour change ${ }^{38}$. In order to better engage a child or adolescent with cancer in physical activity, behaviour change literature leads us to consider the relevance of theories such as the transtheoretical model, self-determination theory and theory of planned behaviour ${ }^{39}$. These models emphasise the importance of people's beliefs, motivation and self-efficacy in bringing about positive changes. Drawing from these theories, physical activity interventions could involve education, barrier identification, goal setting, selfmonitoring techniques, focus on fun and enjoyment and ongoing support and encouragement ${ }^{40}$. This approach considers the child's unique barriers to physical activity and acknowledges the importance of psychological factors in mediating participation in physical activity ${ }^{39,41}$. Examples can be found across both healthy and chronic disease populations ${ }^{42-44}$; such as, the use of activity monitors for motivation and self-monitoring in breast cancer populations ${ }^{45}$.

Interpersonal interyentions focus on targeting social support systems that surround the individual, ${ }^{38}$ and in this setting could focus on the family and peer groups. Parents within this study described feeling overwhelmed in trying to keep up with the many demands placed on them, a common experience in the cancer treatment setting ${ }^{46}$. Assessing parental knowledge, motivation and perceived barriers to physical activity, for example through survey questions derived from the Theory of Planned behaviour ${ }^{47}$, could inform the provision of targeted education and supportive care, and enable parents to be more effective advocates of physical activity. Models of care that emphasise the parental role in physical activity promotion could provide guidance in achieving this. In populations of children with cystic fibrosis, interventions provide consistent messaging regarding the disease-specific benefits of physical activity, and individualised education around how to safely facilitate it. ${ }^{44}$ In line with recommendations made within this study, a potential role for supervised and individualised physical activity sessions is evident. In contrast to interventions driven by therapeutic intent, sessions that focus on fun, child-led physical activity within the family routine, could help to build parent and child confidence and motivation. Sessions may also work towards minimising the disengagement with sport and activity commonly seen in this population. ${ }^{48}$ 
Organisational factors are addressed via interventions targeting health settings, in this case the hospital-or cancer treatment centre. ${ }^{38}$ Organisational culture, priorities and values have a strong influence on physical activity opportunities in other settings. ${ }^{38,49}$ Despite the strong focus on medical management and safety that exists in cancer treatment settings, this focus does not necessarily have to be at the expense of physical activity. Aligning the treating institution's values towards physical activity could inform the development of policy and clinical practice guidelines that emphasise its importance and ensure its safety. A review of policies and practices pertaining to room isolation to align with available literature ${ }^{50,51}$ is an example of a hospital initiative that could facilitate greater participation in physical activity. The development of treatment in the home programs may also be valuable as a means to reducing time spent in the hospital environment. Changes in workplace culture require motivation, leadership, strategy, resources and time ${ }^{52,53}$ and necessitate a collective shift in beliefs, values, behaviours and routines of treating staff. ${ }^{54}$ Many of the changes proposed in this study rely upon funding for professional services and equipment, in addition to a change in mindset in the provision of care. Parents in this study called for a shift in perspective to ensure a holistic approach to their child's care. Positive changes emphasising the importance of physical activity are seen in the adult cancer treatment setting. Rehabilitation using the stepped model of care in adult treatment settings is one example of a changing mindset toward prevention and maximising wellbeing. ${ }^{55}$

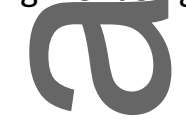

Early literature on physical activity interventions for children during acute cancer treatment focused on exercise prescription strategies that address physical impairments ${ }^{9,56}$. More recently, promising examples of complex intervention design have emerged in the acute paediatric cancer setting, but more work needs to be done to ensure the strategies used are theoretically informed, feasible and address factors beyond the child and family unit ${ }^{18,43,57}$. Based on our results, barriers to physical activity are specific to the individual and can include factors that extend beyond the child. Children and parents also show a limited capacity to overcome these barriers without considerable support and guidance. Moving forward, it is important that physical activity interventions broaden their focus to address environmental and organisational barriers and employ techniques that acknowledge the individual experience of inactivity for each child over the entire course of acute treatment.

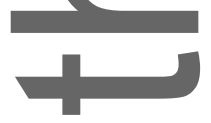

The strength of this study lies in the focus on the parental experience, specifically during acute cancer treatment, providing new perspectives. The theoretical framework of this study is transparent, consistent with the aims and informed the method used. Attaining saturation of data was another factor that strengthened the validity of results. There were potential limitations. The interviewer was a physiotherapist who worked on the cancer ward, this could have potentially biased results due to pre-existing perceptions and opinions regarding the phenomenon under study. Strategies such as member checking and reflexive reporting were imperative to ensure researcher permeability. Efforts were made to recruit parents of children across a wide range of diagnosis groups, yet there were patient populations that remained unrepresented, such as children with 
brain tumour of other solid tumours, so we are uncertain if the emerged themes can be generalised to these groups. Diversity was achieved in other important participant characteristics, and a decision was made regarding data saturation due to the absence of additional themes in the latter stages of recruitment. Future research could explore organisational differences across multiple centres, the experiences of staff within the cancer treatment setting and potential cultural influences. Engagement across the final aspects of the social-ecological model, those pertaining to the community and governing policy makers, could be another area of focus.

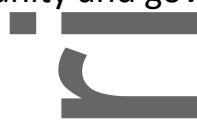

Improving participation in physical activity and reducing the sedentary nature of the hospital environment holds many potential benefits for children with cancer. This study builds on our understanding of the impact of inactivity and the challenges associated with keeping children physically active during acute treatment. Inactivity cannot be addressed by children and parents alone but requires a co-ordinated and multi-faceted approach. The oncology team need to support children to be physically active through their environment and their policies and support must be consistent and ongoing. Results from this study may be used to inform the development of future physical activity interventions that are targeted, effective and feasible.

\section{DATA AVAILABILITY STATEMENT}

The data that support the findings of this study are available on request from the corresponding author. The data are not publicly available due to privacy and ethical restrictions.

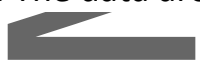

\section{CONFLICT OFINTEREST STATEMENT}

There are no confligts of interest to declare in the production of this paper.

\section{ACKNOWLEDGEMENTS}

This work was supported by an Australian Government Research Training Program Scholarship and Murdoch Children's Research Institute PhD Top-up Scholarship.

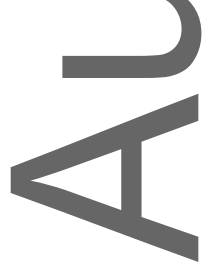




\section{LEGEND}

Table 1 Eligibility criteria

Eligibilit

Table 1 Eligibility criteria

\begin{tabular}{ll}
\hline Inclusion & Exclusion \\
\hline$\quad$ Parent or primary carer of a child: & $\bullet$
\end{tabular}

- $\quad$ Parent or primary carer of a child:

- Not child's primary carer

- Aged 4-18 years

- Diagnosed with cancer or pre-malignant condition

requiring HCT in previous 9 months

- Diagnosed with relapsed disease within previous 9 months

- Receiving or received cancer treatment at $\mathrm{RCH}$

- Mother, father or primary carer of a child:

- $\quad$ Aged $>18$ years or $<4$ years

- $\quad$ Diagnosed with cancer or pre-malignant

condition requiring $\mathrm{HCT}>9$ months ago

- $\quad$ Did not receive cancer treatment at $\mathrm{RCH}$

- Able to undertake interview in English

- Required an interpreter for interview

HCT, hematopoietic cell transplantation; $\mathrm{RCH}$, Royal Children's Hospital
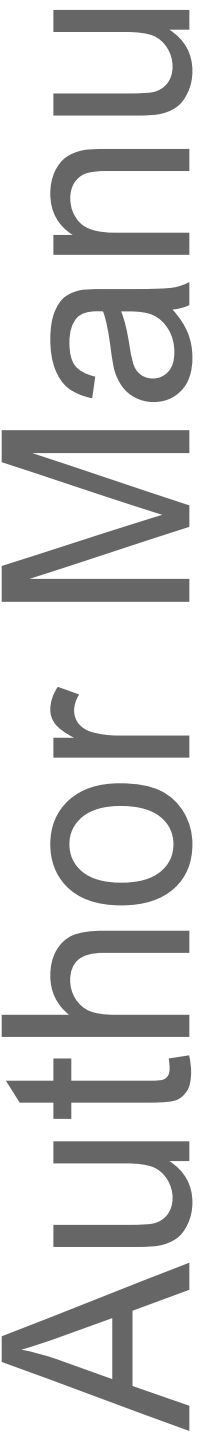

This article is protected by copyright. All rights reserved. 


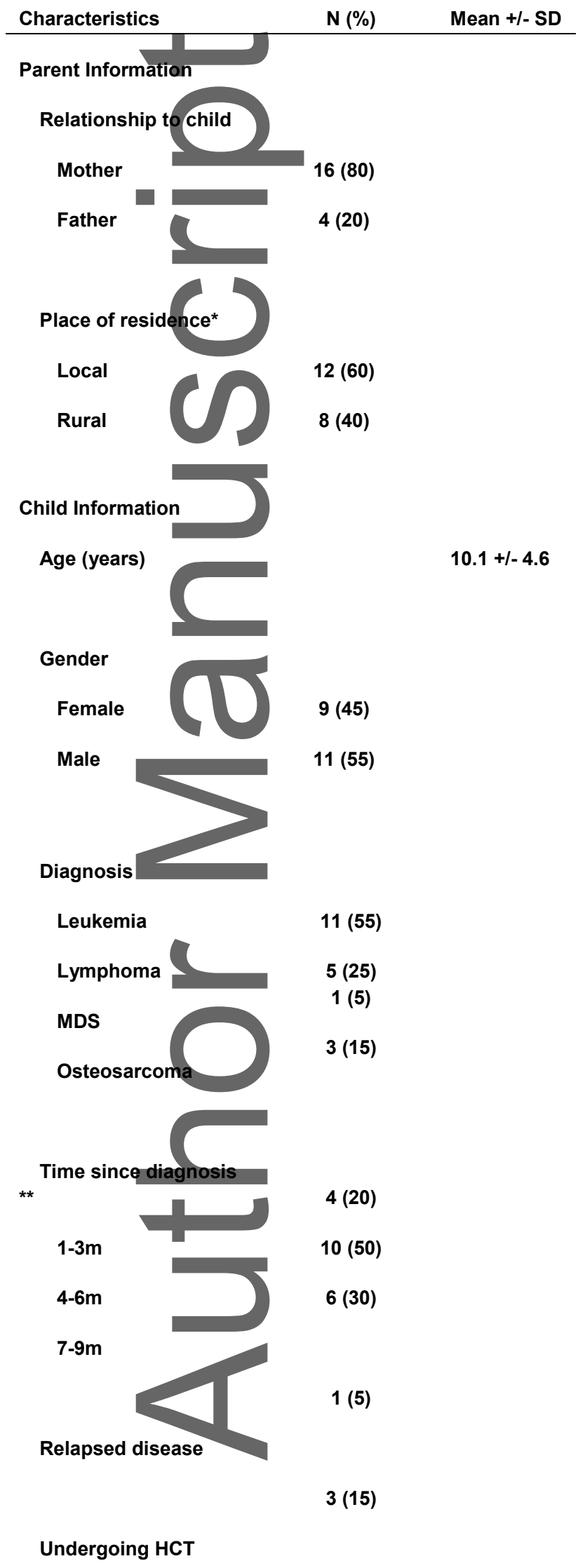

${ }^{*}$ Rural defined as living $>100 \mathrm{~km}$ from hospital

This article is protected by copyright. All rights reserved. 
(Asp

\section{Cancer Diagnosis}
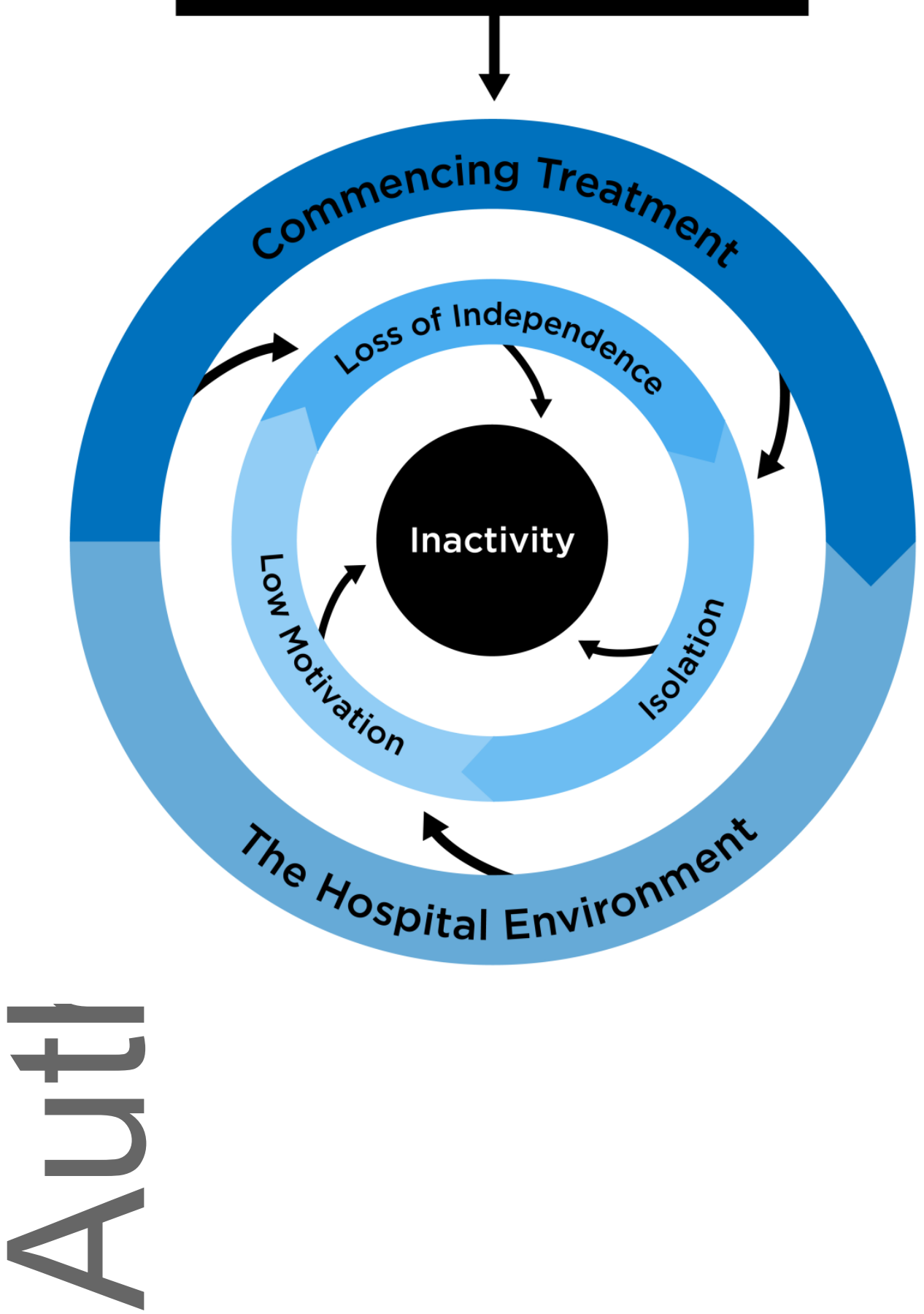


\section{REFERENCES}

1. Aznar S, Webster AL, San Juan AF, et al. Physical activity during treatment in children with leukemia. A pilot study. Appl Physiol Nutr Metab. 2006;31(4):407-413.

2. Fuemmeler BF, Pendzich MK, Clark K, et al. Diet, physical activity, and body composition changes during the first year of treatment for childhood acute leukemia and lymphoma. $J$ Pediatr Hematol Oncol. 2013;35(6):437-443.

3. Winter $C$, Müller $C$, Brandes $M$, et al. Level of activity in children undergoing cancer treatment. Pediatr Blood Cancer. 2009;53(3):438-443.

4. Deisenroth A, Sontgerath R, Schuster AJ, et al. Muscle strength and quality of life in patients with childhood cancer at early phase of primary treatment. Pediatr Hematol Oncol. 2016;33(6):393-407.

5. Elmantaser M, Stewart G, Young D, Duncan R, Gibson B, Ahmed S. Skeletal morbidity in children receiving chemotherapy for acute lymphoblastic leukaemia. Arch Dis Child. 2010:archdischild172528.

6. Lavoie Smith EM, Li L, Chiang C, et al. Patterns and severity of vincristine-induced peripheral neuropathy in children with acute lymphoblastic leukemia. J Peripher Nerv Syst. 2015;20(1).37-46.

7. Thorsteinsson T, Larsen HB, Schmiegelow K, et al. Cardiorespiratory fitness and physical function in children with cancer from diagnosis throughout treatment. BMJ Open Sport \& Exercise Medicine. 2017;3(1).

8. White J, Flohr JA, Winter SS, Vener J, Feinauer LR, Ransdell LB. Potential benefits of physical activity for children with acute lymphoblastic leukaemia. Dev Neurorehabil. 2005;8(1):53-58.

9. Grimshaw SL, Taylor NF, Shields N. The feasibility of physical activity interventions during the intense treatment phase for children and adolescents with cancer: A systematic review. Pediatr Blood Cancer. 2016;63(9):1586-1593.

10. Hartman A, te Winkel $\mathrm{ML}$, van Beek RD, et al. A randomized trial investigating an exercise program to prevent reduction of bone mineral density and impairment of motor performance during treatment for childhood acute lymphoblastic leukemia. Pediatr Blood Cancer. 2009;53(1):64-71.

11. Braam KI, Van der Torre P, Takken T, Veening MA, van Dulmen-den Broeder E, Kaspers GJL. Physical exercise training interventions for children and young adults during and after treatment for childhood cancer. The Cochrane Database of Systematic Reviews. 2013;4:CD008796.

12. Corr AM, Liu W, Bishop M, et al. Feasibility and functional outcomes of children and adolescents undergoing preoperative chemotherapy prior to a limb-sparing procedure or amputation. Rehabilitation Oncology. 2017;35(1):38-45. 
13. Gohar SF, Comito M, Price J, Marchese V. Feasibility and parent satisfaction of a physical therapy intervention program for children with acute lymphoblastic leukemia in the first 6 months of medical treatment. Pediatr Blood Cancer. 2011;56(5):799-804.

14. Winter C, Müller C, Hardes J, Gosheger G, Boos J, Rosenbaum D. The effect of individualized exercise interventions during treatment in pediatric patients with a malignant bone tumor. Supportive Care in Cancer. 2013;21(6):1629-1636.

15. Fiuza-Luces $C$, Padilla JR, Soares-Miranda L, et al. Exercise intervention in pediatric patients with solid tumors: The physical activity in pediatric cancer trial. Med Sci Sports Exercise.

2017;49(2):223-230.

16. Gotte M, Kesting S, Winter C, Rosenbaum D, Boos J. Factors that influence participation in physical activities and exercise in pediatric cancer patients during treatment: A qualitative study. Oncol Res Treat. 2014;37:121.

17. Lam KK, Ho Cheung William L, Ho KY, Chung OK, Chan CF. Factors contributing to the low physical activity level for hong kong chinese children hospitalised with cancer: An exploratory study. Journal of Clinical Nursing. 2017;26(1-2):190-201.

18. Cox $\mathrm{CL}$, Zhu L, Kaste SC, et al. Modifying bone mineral density, physical function, and quality of life in children with acute lymphoblastic leukemia. Pediatr Blood Cancer. 2017.

19. Bar-Mor G, Bar-Tal Y, Krulik T, Zeevi B. Self-efficacy and physical activity in adolescents with trivial, mild, or moderate congenital cardiac malformations. Cardiology in the Young. 2000;10(6):561-566.

20. Golan M. Parents as agents of change in childhood obesity - from research to practice. International Journal of Pediatric Obesity. 2006;1(2):66-76.

21. Young B, Dixon-Woods M, Findlay M, Heney D. Parenting in a crisis: Conceptualising mothers of children with cancer. Soc Sci Med. 2002;55(10):1835-1847.

22. Liamputtong P. Qualitative research methods. 4th ed.. ed. South Melbourne, Vic.: South Melbourne, Vic. : Oxford University Press; 2012.

23. Hamilton $A B$, Finley EP. Qualitative methods in implementation research: An introduction. Psychiatry Res. 2019;280:112516.

24. Lincoln YS, Guba EG. Naturalistic inquiry. Beverly Hills, Calif: Beverly Hills, Calif : Sage Publications; 1985.

25. Caspersen CJ, Powell KE, Christenson GM. Physical activity, exercise, and physical fitness: Definitions and distinctions for health-related research. Public Health Rep. 1985;100(2):126131.

26. Braun V, Clarke V, Terry G, Rohleder P, Lyons A. Thematic analysis. Qualitative Research in Clinical and Health Psychology. 2014:95.

27. Boyatzis RE. Transforming qualitative information: Thematic analysis and code development. Sage; 1998.

28. Flick U. Triangulation in qualitative research. A Companion to Qualitative Research. 2004;3:178-183. 
29. Armstrong D, Gosling A, Weinman J, Marteau T. The place of inter-rater reliability in qualitative research: An empirical study. Sociology. 1997;31(3):597-606.

30. Zade H, Drouhard M, Chinh B, Gan L, Aragon C. Conceptualizing disagreement in qualitative coding. Paper presented at: Proceedings of the $2018 \mathrm{CHI}$ Conference on Human Factors in Computing Systems.

31. Creswell JW.Research design: Qualitative, quantitative, and mixed methods approaches. Sage publications; 2013.

32. Fossey $E$, Harvey $C$, Mcdermott F, Davidson L. Understanding and evaluating qualitative research. Australian and New Zealand Journal of Psychiatry. 2002;36(6):717-732.

33. Krefting L. Rigor in qualitative research: The assessment of trustworthiness. American Journal of Occupational Therapy. 1991;45(3):214-222.

34. O'Reilly M,Parker N. 'Unsatisfactory saturation': A critical exploration of the notion of saturated sample sizes in qualitative research. Qualitative Research. 2013;13(2):190-197.

35. Kerr $C$, Nixon A, Wild D. Assessing and demonstrating data saturation in qualitative inquiry supporting patient-reported outcomes research. Expert Review of Pharmacoeconomics \& Outcomes Research. 2010;10(3):269-281.

36. Brunet J, Taran S, Burke S, Sabiston CM. A qualitative exploration of barriers and motivators to physical activity participation in women treated for breast cancer. Disability and Rehabilitation. 2013;35(24):2038-2045.

37. McLeroy KR, Bibeau D, Steckler A, Glanz K. An ecological perspective on health promotion programs. Health Educ Q. 1988;15(4):351-377.

38. Langille J-LD, Rodgers WM. Exploring the influence of a social ecological model on schoolbased physical activity. Health Education \& Behavior. 2010;37(6):879-894.

39. Rhodes RE, Pfaeffli LA. Mediators of physical activity behaviour change among adult nonclinical populations: A review update. International Journal of Behavioral Nutrition and Physical Activity. 2010;7(1):37.

40. Abraham C, Michie S. A taxonomy of behavior change techniques used in interventions. Health Psychology. 2008;27(3):379.

41. Morris J, Oliver T, Kroll T, MacGillivray S. The importance of psychological and social factors in influencing the uptake and maintenance of physical activity after stroke: A structured review of the empirical literature. Stroke Res Treat. 2012;2012.

42. Greaves C, Sheppard KE, Abraham C, et al. Systematic review of reviews of intervention components associated with increased effectiveness in dietary and physical activity interventions. BMC public health. 2011;11(1):119.

43. Lam KK, Li WH, Chung $O$, et al. An integrated experiential training programme with coaching to promote physical activity, and reduce fatigue among children with cancer: A randomised controlled trial. Patient Education and Counseling. 2018;101(11):1947-1956.

44. Moola FJ, Faulkner GEJ, Schneiderman JE. "Cf chatters": The development of a theoretically informed physical activity intervention for youth with cystic fibrosis. Open J Prev Med.

2011;1(03):109. 
45. Singh B, Spence RR, Sandler CX, Tanner J, Hayes SC. Feasibility and effect of a physical activity counselling session with or without provision of an activity tracker on maintenance of physical activity in women with breast cancer - a randomised controlled trial. J Sci Med Sport. 2019.

46. Gibbins J, Steinhardt K, Beinart H. A systematic review of qualitative studies exploring the experience of parents whose child is diagnosed and treated for cancer. Journal of Pediatric Oncology Nursing. 2012;29(5):253-271.

47. Bassett-Gunter R, Stone R, Jarvis J, Latimer-Cheung A. Motivating parent support for physical activity: The role of framed persuasive messages. Health Education Research. 2017; 32(5):412-422.

48. Ness KK, Leisenring WM, Huang $\mathrm{S}$, et al. Predictors of inactive lifestyle among adult survivors of childhood cancer: A report from the childhood cancer survivor study. Cancer. 2009;115(9):1984-1994.

49. Sallis J, Bauman A, Pratt M. Environmental and policy interventions to promote physical activity. Am J Prev Med. 1998;15(4):379-397.

50. Hayes-Lattin B, Leis JF, Maziarz RT. Isolation in the allogeneic transplant environment: How protective is it? Bone Marrow Transplant. 2005;36(5):373-381.

51. Siegel J, Rhinehart E, Jackson M, Chiarello L. 2007 guideline for isolation precautions: Preventing transmission of infectious agents in health care settings. Appl Physiol Nutr Metab. 2007;35(10 Suppl 2):S65-164.

52. Willis $\mathrm{CD}$, Saul J, Bevan $\mathrm{H}$, et al. Sustaining organizational culture change in health systems. Journal of Health Organization and Management. 2016;30(1):2-30.

53. Scott T, Mannion R, Davies HT, Marshall MN. Implementing culture change in health care: Theory and practice. International Journal for Quality in Health Care. 2003;15(2):111-118.

54. Parmelli E, Flodgren G, Beyer F, Baillie N, Schaafsma ME, Eccles MP. The effectiveness of strategies to change organisational culture to improve healthcare performance: A systematic review. Implementation Science. 2011;6(1):33.

55. Alfano CM, Cheville AL, Mustian K. Developing high-quality cancer rehabilitation programs: A timely need. Am Soc Clin Oncol Educ Book. 2016;35:241-249.

56. Rustler V, Hagerty M, Daeggelmann J, Marjerrison S, Bloch W, Baumann FT. Exercise interventions for patients with pediatric cancer during inpatient acute care: A systematic review of literature. Pediatr Blood Cancer. 2017:19.

57. Götte M, Kesting SV, Gerss J, Rosenbaum D, Boos J. Feasibility and effects of a home-based intervention using activity trackers on achievement of individual goals, quality of life and motor performance in patients with paediatric cancer. BMJ Open Sport \& Exercise Medicine. 2018;4(1). 


\section{University Library}

\section{- M M N E R VA A gateway to Melbourne's research publications}

Minerva Access is the Institutional Repository of The University of Melbourne

Author/s:

Grimshaw, SL;Taylor, NF;Mechinaud, F;Conyers, R;Shields, N

Title:

Physical activity for children undergoing acute cancer treatment: A qualitative study of parental perspectives.

Date:

2020-06

Citation:

Grimshaw, S. L., Taylor, N. F., Mechinaud, F., Conyers, R. \& Shields, N. (2020). Physical activity for children undergoing acute cancer treatment: A qualitative study of parental perspectives.. Pediatr Blood Cancer, 67 (6), pp.e28264-. https://doi.org/10.1002/pbc.28264.

Persistent Link:

http://hdl.handle.net/11343/275613 